\title{
Análisis de un modelo integrador del compromiso escolar: relaciones entre variables situacionales, escolares, sociales y personales en alumnos de nivel primario de educación
}

\author{
Analysis of an Integrative Model of School Commitment: \\ Relationship among Situational, School, Social and Personal \\ Variables in Primary School Students
}

\author{
Daiana Yamila Rigo* \\ Instituto de Investigaciones Sociales, Territoriales y Educativas, Consejo Nacional de \\ Investigaciones Científicas y Técnicas, Buenos Aires, Argentina \\ Universidad Nacional de Río Cuarto, Córdoba, Argentina \\ ORCID: https://orcid.org/0000-0003-0312-6429 \\ Danilo Donolo \\ Consejo Nacional de Investigaciones Científicas y Técnicas, Buenos Aires, Argentina \\ Universidad Nacional de Río Cuarto, Córdoba, Argentina \\ ORCID: https://orcid.org/0000-0002-0584-7492
}

Recibido 07-05-19 Revisado 14-08-19 Aprobado 29-10-19 En línea 07-11-19

*Correspondencia

Email: daianarigo@hotmail.com
Citar como:

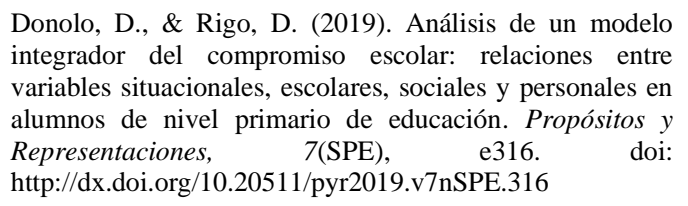

Donolo, D., \& Rigo, D. (2019). Análisis de un modelo integrador del compromiso escolar: relaciones entre alumnos de nivel primario de educación. Propósitos y http://dx.doi.org/10.20511/pyr2019.v7nSPE.316 


\section{Resumen}

El estudio pretende analizar el rol predictivo de la percepción que los estudiantes tienen acerca de las variables situacionales, escolares, sociales y personales de la clase sobre el compromiso escolar afectivo, conductual y cognitivo hacia las tareas escolares. La investigación se desarrolló con 219 alumnos del nivel primario de educación, de escuelas públicas de Argentina. Para la recolección de datos se utilizaron cuatro instrumentos estandarizados -Escala de Compromiso hacia las Tareas Escolares en las Clases del Nivel Primario; Inventario de Percepción del Contexto Educacional como Motivador; Cuestionario de Implicación con el Centro Escolar y, Escala de Orientación Motivacional Intrínseco versus Extrínseca en el Aula- y el registro de las faltas a lo largo del año lectivo, y se solicitó el asentimiento informado. El modelo evaluado, mediante Path Analysis, presenta un buen ajuste a los datos $\left(\mathrm{X}^{2}=20.24, \mathrm{gl}=7, \mathrm{p}=.005, \mathrm{X}^{2} / \mathrm{gl}=2.89\right.$, CFI=.99, $\mathrm{GFI}=.99$, RMSEA=.093-IC 90\%=.047/.14-) y confirma la hipótesis, es decir, que el compromiso afectivo, conductual y cognitivo se ve favorecido por la configuración de la clase y los apoyos obtenidos de pares y docentes, mientras que las creencias personales median la inasistencia, como variables escolar, que influye negativamente sobre cada dimensión de la implicación del estudiante.

Palabras clave: Compromiso; Factores personales; Contexto escolar; Apoyo social; Desvinculación.

\section{Summary}

The study aims at analyzing the predictive role of students' perception about the class's situational, school, social, and personal variables on the affective, behavioral, and cognitive engagement towards school tasks. The research was developed with 219 students of the primary education level from public schools in Argentina. For data collection, four standardized instruments were used: -Scale of Engagement towards School Tasks in Primary Level Classes; Inventory of the Perception of Educational Context as motivator; Questionnaire of Involvement with the School Center and Intrinsic versus Extrinsic Motivational Orientation Scale in the Classroom- as well as the absence record keeping throughout the school year. Furthermore, informed consent was also requested. The model evaluated through Path Analysis presents a good fit to the data (chi square $=20.24, \mathrm{df}=7, \mathrm{p}=.005$, chi square $/ \mathrm{df}=2.89, \mathrm{CFI}=.99, \mathrm{GFI}=.99, \mathrm{RMSEA}=.093-\mathrm{CI}$ $90 \%=.047 / .14-$ ), and confirms the hypothesis, i.e., that the affective, behavioral and cognitive engagement is favored by the configuration of the class and the support from peers and teachers, while personal beliefs mediate absence as school variables that exert a negative influence on every dimension of student involvement.

Keywords: Engagement, Personal Factors, School Context, Social Support, Disengagement.

\section{Introducción}

La desvinculación escolar ha demostrado ser un problema educacional a escala internacional, y a nivel nacional que también preocupa. Recientemente, un estudio sobre los factores endógenos asociados a los sistemas escolares relacionados con la desconexión escolar en Latinoamérica y el Caribe, muestra que entre el $30 \%$ y el $43 \%$ de los estudiantes que abandonaron la escuela señalaron motivos como, el aburrimiento, la falta de interés en la escuela o el estudio, el fracaso, y la dificultad para entender las explicaciones de los maestros o el contenido escolar (CAF, 2018), como comportamientos ligados a la definición del compromiso escolar. Problemáticas sobre la cual varios actores y escenarios tienen algún grado de participación en su promoción (Rigo \& Donolo, 2018a). La calidad del contexto educativo, los apoyos sociales y escolares recibidos, así como, los factores más intrínsecos influyen en la manera en cómo los estudiantes se sienten implicados con el hacer en la escuela. 
Lo cierto, es que, los estudios internacionales se han centrado en la franja etaria de la adolescencia, cubriendo el nivel medio y superior de educación, no considerando los escenarios educativos que abarcan el nivel primario de educación (NSSE, 2018; OCDE, 2017). Con el propósito de profundizar sobre las primeras etapas de escolarización, aparece como novedoso y necesario estudiar los factores contextuales y personales que tienen un rol predictivo sobre la continua vinculación-desvinculación escolar en estudiantes que cursan la escuela primaria, para tender a futuro prácticas educativas que ayuden a diseñar entornos educativos que propicien el compromiso.

Asimismo, el creciente interés por estudiar el compromiso en los últimos años se relaciona a su carácter maleable y modificable (Fredricks, Blumenfeld \& Paris, 2004); postulado que se sustenta en dos grandes perspectivas teóricas, el modelo contextual de Lam, Wong Yang y Liu (2012) y el enfoque de la complejidad contextual de Shernoff, Ruzek y Sinha (2016); considerando ambos postulados y estableciendo que una parte importante de los aprendizajes escolares se dan en torno a las tareas que resuelven los estudiantes en clase, como situación donde los alumnos manifiestan una multiplicidad de comportamientos relacionadas con su compromiso en función de variables internas y externas a dicha situación.

\section{Compromiso en el contexto escolar}

La manifestación más visible de un estudiante implicado en y con las tareas de aprendizaje se registra cuando se mantiene atento, participa y su muestra interesado por aquello que acontece en torno al desarrollo de la clase. No obstante, tales indicadores, no bastan para definir un constructo que excede las expresiones comportamentales y afectivas incluyendo además estrategias cognitivas y de autorregulación como procesos más internos y difíciles de reconocer a simple vista.

Esta complejidad inherente al término y su concepto se recoge en las definiciones que lo enmarcan como un meta-constructo, integrado por tres dimensiones -afectiva, conductual y cognitiva- que suponen una energía en acción que denota que los alumnos están implicados, conectados y comprometidos activamente para aprender y rendir, en contraste con la participación superficial, la apatía o la falta de interés. Más concretamente, el compromiso hacia las tareas escolares se caracteriza por la intensidad y la emoción con la cual los estudiantes se involucran para iniciar, llevar a cabo y finalizar las actividades de aprendizaje (Appleton, Cristenson, Kin \& Reschly, 2006; Rigo, 2017). Asimismo, de manera más reciente las aproximaciones conceptuales lo enmarcan como un comportamiento que dista de ser estable, moldeado por las acciones y decisiones que los maestros toman como parte de la planificación del diseño instructivo, y las que adoptan los estudiantes como manera de influir en la definición de esa propuesta curricular expresando inclinaciones y afinidades.

Las manifestaciones afectivas se asocian con el interés y la atención que los estudiantes sienten y perciben cuando desarrollan las actividades escolares, en su contraste más apuesto encontramos la apatía y abulia hacia las tareas desarrolladas como vehículos de nuevos aprendizajes. Lo conductual se relaciona con aquellas expresiones que tienen lugar dentro del aula cuando se producen debates o intercambios, la participación aparece como un indicador clave, junto a respuestas activas frente a solicitudes de maestros o compañeros para poder avanzar en la resolución de la práctica que se esté llevando a cabo dentro del ala. Finalmente, lo cognitivo se refiere al uso de estrategias de aprendizaje que puedan definirse en el continuo superficiales profundas, así como, aquellas orientadas a revisar, monitorear y reflexionar sobre cómo se llevan a cabo los aprendizajes (Rigo, 2017; Rigo \& Donolo, 2018b). Estas tres dimensiones, reaccionan más a la configuración del contexto instructivo, a diferencia de la reciente incorporación bajo la denominación de agencia, de comportamientos más proactivas y transaccionales, en tanto los estudiantes orientan acciones para modificar el contexto de aula como eje central para vincularse haciendo explícitos sus intereses, preferencias y gustos (Reeve, 2013). 
Si bien los estudios empíricos han avanzado considerablemente, los procesos de desvinculación aún siguen siendo una alerta que mantiene a los educadores, y a los teóricos, en la buscada de nuevas formas de reenganchar a los alumnos con los procesos de aprendizajes formales. En este sentido, seguir investigando aquellos factores que ayudan a predecir el compromiso estudiantil es clave para definir a futuro prácticas para promoverlo.

\section{Factores internos y externos al compromiso}

Los primeros estudios sobre la implicación escolar centraron su mirada hacia los factores intrínsecos al individuo, a la fecha, los resultados de diversos estudios aseveran que no es suficiente mirar solo aquello que tiene lugar en el sujeto como agente activo de sus aprendizajes, sino que dentro de la definición de la zona de desarrollo próxima definida por Vygotsky y de la mano de los postulados socio-culturales se reconoce que los artefactos de la cultura median, influyen y tienen algún grado de injerencia en cómo los estudiantes se comprometen con las tareas escolares (Vigotsky, 1988).

Si partimos de considerar al aprendizaje como un proceso eminentemente social, el sostén de los otros y el rol que tienen en los procesos de implicación es clave. Shernoff (2013) plantea que los factores ligados al sujeto son lo que menos peso tienen para explicar los procesos de aprendizaje, no obstante, son las variables del contexto instructivo las que marcan la diferencias en el compromiso manifestado por los estudiantes.

Considerar la compleja red conceptual que implica estar comprometidos en los procesos de enseñanza-aprendizaje, invita a contemplar una multiplicidad de factores que tienen que ver con rasgos internos y externos al estudiante. Con ese propósito, el presente trabajo investiga el papel predictivo que variables personales, escolares, situaciones y sociales tienen el compromiso afectivo, conductual y cognitivo expresado por los estudiantes de nivel primario de educación.

A la actualidad se pueden identificar dos modelos que expanden la comprensión del compromiso, por un lado, el contextual de Lam et al. (2012) y, por el otro, el de complejidad ambiental de Shernoff et al. (2016). Ambos destacan el rol de la configuración de la clase como factor elemental de la participación de los estudiantes, mediada por dimensiones de apoyo social e intrínsecos al estudiante, como las creencias motivacionales.

Contemplando el modelo de Lam et al. (2012) el compromiso de los estudiantes queda delimitado en una red conceptual que revela que los estudiantes se implican en la escuela cuando sienten que sus maestros adoptaron prácticas instruccionales motivantes -desafiantes, con valor de utilidad, orientadas a promover la curiosidad y la autonomía- y reciben apoyo socioemocional de sus maestros, padres y compañeros. Asimismo, la participación de los alumnos es más alta cuando tienen objetivos de aprendizaje claros, alto sentido de autoeficacia, y atribuciones sustentadas en el esfuerzo (Liu et. al., 2018). Lo más importante es que, los estudiantes que participaban en las escuelas, con frecuencia experimentan emociones positivas y sus maestros valoran y reconocen sus comportamientos, acciones y pensamientos.

Retomando los aportes de Shernoff et al. (2016), el modelo de complejidad contextual entiende que la calidad del entorno de aprendizaje se conceptualiza en términos de complejidad ambiental, o la presencia simultánea de retos y apoyos contextuales. El desafío ambiental se refiere a los retos, tareas, actividades, metas, estructuras y expectativas destinadas a guiar la acción o el pensamiento de los estudiantes; mientras que, el apoyo ambiental se refiere a los recursos instrumentales, sociales y emocionales disponibles para ayudar a los estudiantes a alcanzar los desafíos formulados.

En ninguno de los modelos anteriores, la asistencia a la escuela se contempla como una variable que posiblemente pueda mediar en el compromiso de los estudiantes. La mayoría de las investigaciones se desarrollan por sobre todo en el nivel superior de educación, mostrando 
evidencia aún ambivalente. La revisión realizada por Stoner y Fincham (2012) pone en evidencia que, si bien la tendencia es asumir que la inasistencia se traduce en resultados educativos disminuidos, algunos estudios la asocian a una disminución de las expectativas de los estudiantes hacia los profesores o al mayor acceso de los estudiantes a la información a través de los avances tecnológicos. Lo cierto es que, tales resultados son relativos al ámbito universitario y, en lo que respecta específicamente al nivel primario de educación, el informe de la CAF (2018), revela que la inasistencia se correlaciona con la desvinculación escolar.

Basándonos en los modelos expuestos y sumando las primeras evidencias sobre la importancia de atender a la inasistencia, la presente investigación se orienta a definir un modelo explicativo del compromiso escolar orientado hacia las tareas de aprendizaje que llevan a cabo los estudiantes del nivel primario de educación, a partir del análisis de variables que refieren tanto a competentes situaciones, como la configuración de la clase percibida por los alumnos; sociales, como la percepción del apoyo recibido por pares y maestros; escolares, como la frecuencia de faltas en el año lectivo y; personales, circunscriptas en las creencias motivacionales desarrollados por los niños respecto a metas, atribuciones y sentido de autoeficacia.

Específicamente, el trabajo evalúa dimensiones de la configuración de la clase percibidas por los alumnos (desafío, curiosidad, valor de utilidad, autonomía, reconocimiento y evaluación), aspectos de las creencias motivacionales de los estudiantes como rasgos personales (atribuciones, sentido de autoeficacia y orientación a metas), componentes del apoyo social percibido (compañeros y maestros), la inasistencia como variable escolar, y tres componentes del compromiso (afectivo, conductual y cognitivo).

En función de la bibliografía revisada se espera encontrar sustento para el siguiente modelo hipotetizado de relaciones entre variables (figura 1), es decir, (a) que la percepción de la configuración de la clase y el apoyo social predigan positivamente el compromiso de los estudiantes, y (b) que las creencias motivacionales de los estudiantes y los apoyos socialesáulicos percibidos tengan un efecto mediador negativo sobre cada dimensión del compromiso a través de la inasistencia.

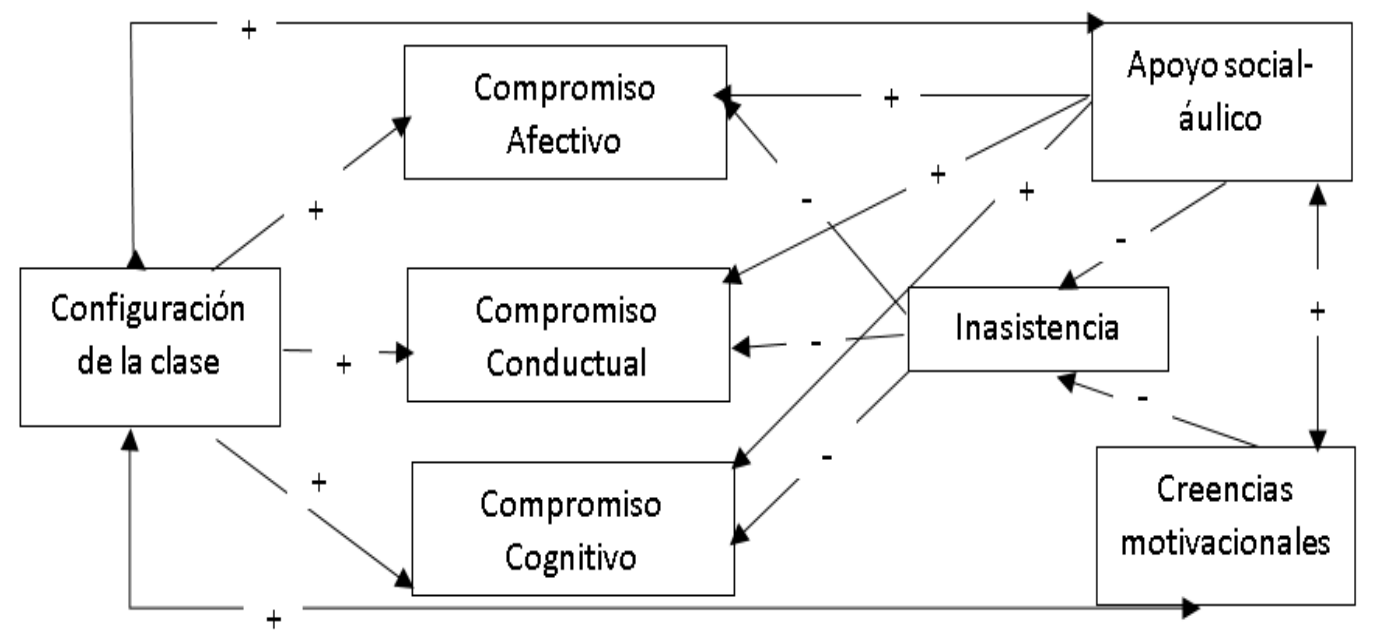

Figura 1. Modelo explicativo hipotetizado del compromiso escolar

Esta investigación presenta aportes novedosos no contemplados por la literatura y estudios previos; analiza conjuntamente variables que definen al contexto situacional, social y personal de la clase en estudiantes de nivel primario de educación para predecir el compromiso escolar, asimismo, examina una variable escolar poco estudiada como la inasistencia a clase, decisiva para comprender el compromiso escolar de los estudiantes hacia las tareas escolares y planificar acciones tendientes a promoverlo. 
El objetivo del estudio fue identificar el rol que cumplen algunas variables situacionales, sociales, personales y escolares sobre el compromiso escolar afectivo, conductual y cognitivo del estudiante hacia las tareas de aprendizaje. Se desarrolló un estudio transversal, correlacional y explicativo (Sampieri, Collado \& Lucio, 2010).

\section{Método}

\section{Participantes}

La muestra estuvo conformada por 219 estudiantes ( $49 \%$ mujeres; $M=10.41$ años, $\mathrm{DE}=1,29$ ) que estaban cursando el segundo ciclo de la educación primaria $\left(4^{\text {to }}-31 \%-, 5^{\text {to }}-33 \%-\right.$ o $6^{\text {to }}-36 \%$ grado) de escuelas públicas de una provincia ubicada al centro de Argentina. Se optó por un muestreo por conveniencia, en tanto se accedió a las escuelas, cuyos directivos y su receptiva inspección, autorizaron el ingreso. La investigación propuesta se inscribe en un proyecto mayor de la escuela para el mejoramiento y compromiso de los alumnos para sus resultados escolares.

\section{Instrumentos}

Para la recolección de datos se utilizaron distintos instrumentos para atender a cada una de las variables en estudio. A continuación, se describen en detalle.

Compromiso hacia las tareas escolares. Se evaluaron las percepciones estudiantiles sobre las dimensiones afectivas, conductuales y cognitivos de implicación a través de la Escala de Compromiso hacia las Tareas Escolares en las Clases del Nivel Primario. Los alumnos puntuaron la presencia de cada comportamiento utilizando una escala de tipo Likert de 5 puntos. La escala presenta adecuadas propiedades psicométricas, indicando una estructura factorial tridimensional -afectiva, conductual y cognitiva- y una aceptable consistencia interna (Rigo \& Donolo, 2018b).

Configuración de la clase, como variable situacional. Se usó el Inventario Percepción del Contexto Educacional como Motivador de 24 ítems (Lam, Pak \& Ma, 2007) adaptada para su uso en población bajo estudio. El instrumento valora 6 dimensiones del contexto instructivo, a saber: desafío, valor de utilidad, curiosidad, autonomía, reconocimiento y evaluación. Los estudiantes puntuaron la ocurrencia de cada comportamiento utilizando una escala de 5 puntos. Para la presente investigación se analizó la estructura interna mediante análisis factorial confirmatorio y coeficiente alpha de Cronbach, encontrando resultados aceptables para una estructura unidimensional $\left(X^{2}=21.89, \mathrm{p}=.0009, X^{2} / \mathrm{gl}=2.43, \quad \mathrm{CFI}=.97, \mathrm{GFI}=.98, \quad \mathrm{RMSEA}=.079\right.$-IC $90 \%=.037 / .12-, \square=.86)$.

Apoyo social percibido, como variable social. Se empleó el Cuestionario de Implicación con el Centro Escolar desarrollado por Ros (2009), para indagar el apoyo recibido de compañeros y maestros, a través de 12 ítems. Los estudiantes usaron una escala de 5 puntos para valorar las relaciones establecidas con cada agente consultado. Un análisis factorial confirmatorio para esta investigación reveló resultados óptimos para una estructura unidimensional $\left(X^{2}=97.3, \mathrm{p}=.0001\right.$, $\left.X^{2} / \mathrm{gl}=1.8, \mathrm{CFI}=.88, \mathrm{GFI}=.94, \mathrm{RMSE}=.057-\mathrm{IC} 90 \%=.038 / .074-\right)$. El análisis de consistencia interna arrojó un valor adecuado de $\square=.80$.

Creencias motivacionales, como variable personal. Se utilizó la Escala de Orientación Motivacional Intrínseco versus Extrínseca en el Aula de Harter (1980), adaptada al español por González-Torres y Tourón (1994). Se contemplaron 18 ítems para valorar las creencias motivacionales, que referían a Preferencia por el reto vs. Preferencia por el trabajo fácil; Curiosidad e interés vs. Agradar al maestro y obtener calificaciones; Criterios de éxito y fracaso internos vs. Criterios externos. Los estudiantes las estimaron considerando una escala con valores que oscilaban entre 1 y 4 . Para la presente investigación, el AFC reveló una estructura unidimensional $\left(X^{2}=363.7, \quad \mathrm{p}=.000, \quad X^{2} / \mathrm{gl}=2.68, \quad \mathrm{CFI}=.87, \quad \mathrm{GFI}=.89, \quad \mathrm{RMSE}=.089-\mathrm{IC}\right.$ $90 \%=.078 / .100-)$, con adecuada consistencia interna $\square=.78$. 
Inasistencia, como variable escolar. Se solicitaron a los maestros el total de inasistencia que los estudiantes tuvieron a lo largo del ciclo lectivo, producto de la sumatoria de las faltas acumuladas durante los tres semestres, tal cual fueron notificadas en las libretas de calificaciones al finalizar el año escolar.

\section{Procedimiento}

En primer lugar, se contactó con los directores de las escuelas públicas para informarles sobre los objetivos de la investigación, luego se solicitó la autorización a la inspección de escuelas primarias de la Provincia de Córdoba-Argentina, para ingresar a los establecimientos educativos. En segundo lugar, se solicitaron los correspondientes asentimientos y consentimientos informados, a los padres de los estudiantes y a los docentes para acceder a las aulas, respectivamente; resguardando la identidad y el anonimato de las respuestas ofrecidas.

Las escalas se administraron en horario escolar bajo la presencia del maestro a cargo de la clase, durante cuatro encuentros de una hora cada uno. Desde la dirección de cada escuela autorizó el acceso a las inasistencias de cada estudiante a lo largo del ciclo lectivo.

\section{Análisis de datos}

Para las escalas se calcularon los puntajes totales sumando las respuestas brindadas para cada ítem. Se realizó un path analysis, siguiendo los criterios y etapas establecidas por Pérez, Medrano y Sanchéz Rosas (2013) para evaluar el ajuste del modelo teórico propuesto, los efectos directos e indirectos, así como los coeficientes path significativos y el porcentaje de la varianza explicada. Se contemplaron diversos índices de ajuste, a saber; $X^{2}$ y $X^{2} / g l$, CFI -índice de ajuste comparado, GFI -índice de bondad de ajuste y RMSEA -error cuadrático medio de aproximación-, tomando como referencia los valores y pautas de interpretación de Pérez et al. (2013) y, Rigo y Donolo (2019). Los datos fueron cargados y procesados con el programa IBM SPSS y AMOS 16.

\section{Resultados}

Se evaluó el ajuste del modelo propuesto y se obtuvieron valores aceptables $\left(X^{2}=20.24, \mathrm{gl}=7\right.$, $\mathrm{p}=.005, X^{2} / \mathrm{gl}=2.89, \mathrm{CFI}=.99, \mathrm{GFI}=.99$, RMSEA=.093-IC 90\%=.047/.14-). Asimismo, se encuentra que la varianza explicada $\left(\mathrm{R}^{2}\right)$ tiene un rico poder explicativo, lo que se refleja en los coeficientes de determinación disponibles en la Figura 2, a saber, de un $82 \%$ para el compromiso afectivo, de un $79 \%$ para la dimensión conductual y de un $86 \%$ para la cognitiva, y significativos a un p-valor $<.01$, significancia que también se presenta para cada coeficiente path. 


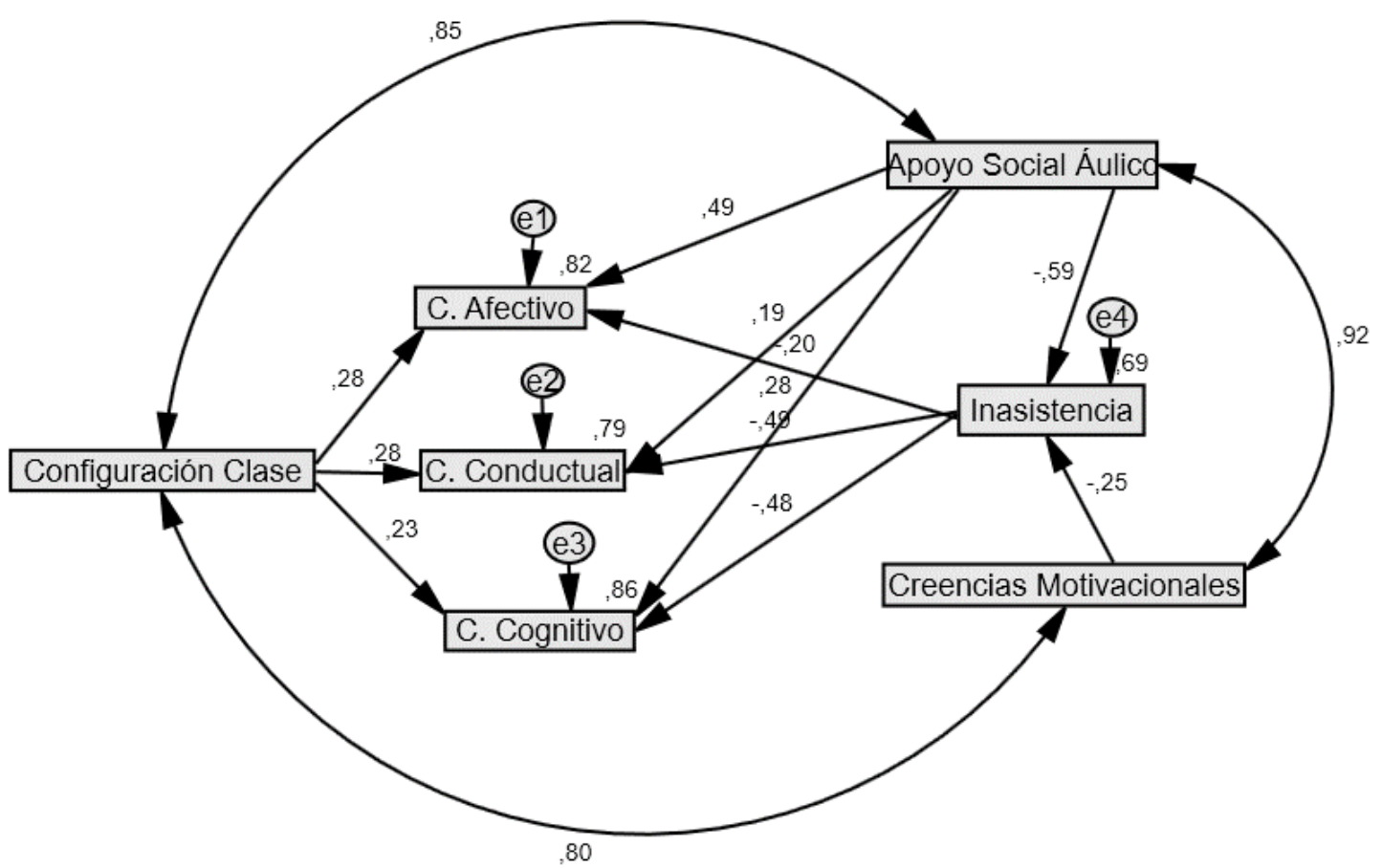

Figura 2. Relaciones estructurales entre variables (coeficientes path estandarizados)

En el modelo se constata que la configuración de la clase predijo positivamente cada dimensión del compromiso reportado por los estudiantes, así como, la percepción del apoyo social-áulico que, a la vez, incluye negativamente sobre la inasistencia. La cantidad de faltas a la institución educativa se ve influenciada negativa y directamente por la variable social de apoyos recibidos en el aula por parte de docentes y alumnos y por las creencias motivacionales de los estudiantes que, impactan indirectamente y también negativamente sobre las dimensiones de compromiso afectiva, conductual y cognitiva (tabla 1).

\section{Tabla 1}

Coeficiente path ( $\beta)$, efectos estandarizados totales, directos e indirectos entre variables

\begin{tabular}{lccc}
\hline \multicolumn{1}{c}{ Variables } & Efecto total & $\begin{array}{c}\text { Efecto } \\
\text { directo }\end{array}$ & $\begin{array}{c}\text { Efecto } \\
\text { indirecto }\end{array}$ \\
\hline Inasistencia - Creencias motivacionales & -.249 & -.249 & \\
Inasistencia - Apoyo Social áulico & -.593 & -.593 & \\
C. Conductual - Creencias motivacionales & .121 & & .121 \\
C. Conductual - Apoyo Social áulico & .479 & .191 & .288 \\
C. Conductual - Configuración Clase & .284 & .284 & \\
C. Conductual - Inasistencia & -.486 & -.486 & \\
C. Cognitivo - Creencias motivacionales & .120 & & .120 \\
C. Cognitivo - Apoyo Social áulico & .568 & .284 & .284 \\
\hline
\end{tabular}




\begin{tabular}{llll}
\hline C. Cognitivo - Configuración Clase & .235 & .235 & \\
C. Cognitivo - Inasistencia & -.480 & -.197 & .049 \\
C. Afectivo - Creencias motivacionales & .049 & & .117 \\
C. Afectivo - Apoyo Social áulico & .609 & .492 & \\
C. Afectivo - Configuración Clase & .276 & .276 & \\
C. Afectivo - Inasistencia & -.197 & -.197 & \\
\hline
\end{tabular}

Nota: todos los valores son significativos $(\mathrm{p}<.01)$.

\section{Discusión}

En el presente estudio se confirman resultados nuestros y de otros estudios, sumándose nuevos aportes que ayudan a comprender las relaciones entre las variables estudiadas. En primera instancia, tal como se esperaba la configuración de la clase predice positivamente el compromiso afectivo $(\beta=.28)$, conductual $(\beta=.28)$ y cognitivo $(\beta=.23)$ de los estudiantes. Según diversos estudios, la interrelación entre las dimensiones de la clase favorece en los estudiantes estar más implicados, ya sea interesados por la materia y las actividades escolares, más participativos en los debates y abiertos a responder preguntas o realizar consultar, como a regular sus procesos de aprendizaje (Ames, 1992; Blumenfeld, Kempler \& Krajcik, 2006; Lam et al., 2012; Nichols \& Dawson, 2012; Rigo \& Donolo, 2014; Shernoff, 2013; Stipek, 1996). Específicamente, tales investigaciones marcan que proponer tareas desafiantes, con valor de utilidad e interesantes, junto a intercambios orientados a redefinir y monitorear el trabajo áulico, son dimensiones que favorecen el compromiso de los estudiantes.

En segunda instancia, la colaboración entre pares y maestros dentro del aula $(\beta=-.59)$ y las creencias motivacionales $(\beta=-.25)$ desarrolladas por los estudiantes, tal como se hipotetizada tienen un rol predictivo y claramente negativo sobre la inasistencia. Esto implicaría que cuando los estudiantes tienen una fuerte creencia en sus propias capacidades para desempeñarse en las tareas de aprendizaje, pero a la vez reciben adecuados soportes de sus compañeros y docentes, la cantidad de inasistencia son menores. En relación a los factores personales, los datos encontrados son coincidentes con lo expuesto por informe CAF (2018), donde las bajas expectativas personales se asocian a tasas bajas de asistencia escolar y de desvinculación, tendencia que, se asienta también en la percepción que los estudiantes construyen sobre la configuración de la clase (Rigo, 2017).

Asimismo, los resultados muestran que el apoyo social es clave para potenciar las creencias motivacionales de los estudiantes, de allí el impacto sobre las faltas acumuladas durante el año lectivo por los estudiantes. En tal sentido, estudios previos son más claros con respecto a la vinculación del compromiso con el apoyo social percibido (Gutiérrez, Tomás, Romero \& Barrica, 2017) que, a la inasistencia (Stoner \& Fincham, 2012; Lukkarinena, Koivukangas \& Seppälä, 2016; Miranda-Zapata, Lara, Navarro, Saracostti \& de-Toro, 2018). No obstante, los resultados encontrados muestran la importancia que tiene el contexto social y personal para promover la implicación de los estudiantes y la asistencia a clases. En tal sentido, en futuros estudios se deberá indagar en mayor profundidad el papel que ambas variables tienen sobre la deserción escolar, siendo un tema que preocupa en la agenda educativa actual, nacional e internacional. Al respecto, Glasman (2000) menciona dos tipos de desvinculación, una asociada a estudiantes que abandonan los estudios tempranamente, y otra relacionada con los estudiantes que se desconectan sin moverse de su sitio, es decir, sin abandonar los estudios, cuyos motivos están ligados a no romper con lo que, dentro del entorno cercano, sigue siendo lo esperado según las expectativas construidas. 
En tercera instancia, se confirma el rol predictivo de la inasistencia en el nivel primario de educación y en el sentido esperado, al encontrar que el número de ausencias a clases es un buen estimador de los procesos de implicación de los estudiantes, en tal sentido, los resultados muestran una influencia negativa sobre las tres dimensiones del compromiso hacia las tareas académicas, afectivo $(\beta=-.20)$, conductual $(\beta=-.49)$ y cognitivo $(\beta=-.48)$. Los datos coinciden con las tendencias marcadas por Stoner y Fincham (2012), quienes identifican que, los alumnos que asisten a clase presentan una mayor implicación y deseo de atender a las explicaciones de los docentes, en tanto contemplan que proporcionan información y tareas relevantes e interesantes para avanzar en sus estudios. Asimismo, Van Blerkom (2012), en el sentido de lo encontrado en este estudio, detalla en su investigación que la inasistencia tiende ser uno de los motivos más frecuentes que mencionan los estudiantes que perciben a las clases como aburridas.

En cuarta instancia, el apoyo social predijo también los comportamientos afectivos $(\beta=.49)$, conductuales $(\beta=.19)$ y cognitivos $(\beta=.28)$ de los estudiantes, dimensión que covaria junto con la configuración de la clase. Estudios anteriores muestran que los apoyos sociales percibidos por los estudiantes predicen el compromiso escolar incluidas las variables del entorno áulico, resaltando que los maestros y los compañeros ejercen una mayor influencia en la implicación que el apoyo familiar, además exponen que los aspectos del apoyo docente autonomía y estructura- y del apoyo entre pares -académico y emocional- intervienen positivamente sobre el grado de participación e involucramiento de los alumnos (Brewster \& Bowen, 2004; Garcia-Reid, Reid \& Peterson, 2005; Kiefer, Alley \& Ellerbrock, 2005; Strati, Schmidt \& Maier, 2017).

En síntesis, el presente trabajo intentó aportar a la definición del compromiso del estudiante con las tareas escolares como constructo complejo, maleable y dependiente de una multiplicidad de factores que exceden a los personales y envuelven algunas dimensiones asociadas al contexto de la clase. En ese marco se estableció un modelo explicativo sustentado en variables situacionales, sociales, escolares e internas al estudiante, comprobando el impacto y la capacidad predictiva que tienen para comprender la implicación en el nivel primario de educación. Los resultados muestran ser susceptibles de atender a planteos prácticos dentro de las instituciones escolares y las aulas, a fin de atender problemáticas de desvinculación que tiene una fuerte incidencia, ya desde los primeros años de escolarización (Fredricks, Reschly \& Christenson, 2019; Rigo \& Donolo, 2018). Asimismo, lo encontrado reafirma la importancia de seguir generando modificaciones en las formas de diseñar la clase y las tareas escolares, como elemento situacional clave para estudiantes más implicados en lo escolar; no obstante, también se revela la relevancia de atender de manera sostenida a las relaciones interpersonales que afianzan el interés por aprender y asistir al colegio, lo que a la vez hace a estudiantes más motivados, autónomos y dispuestos a asumir desafíos educacionales.

Finalmente, con respecto a las limitaciones, en futuras investigaciones el auto reporte como instrumento central de recolección precisa medidas complementarias como el registro de comportamientos en el aula, a partir de observaciones estructuradas (Fredricks \& McColskey, 2012); asimismo, se considera necesario trabajar con una muestra representativa para poder extrapolar los resultados a la población objeto de estudio, dada la riqueza de los resultados encontrados y el potencial impacto para transformar las prácticas educativas.

\section{Referencias}

Ames, C. (1992). Classroom: Goals, structures, and student motivation. Journal of Educational Psychology, 84(3), 261-271.

Appleton, J., Christenson, S., Kin, D., \& Reschly, A. (2006). Measuring cognitive and psychological engagement: validation of the student engagement instrument. Journal of School Psychology, 44, 427-445. doi: https://doi.org/10.1016/j.jsp.2006.04.002 
Blumenfeld, P., Kempler, T., \& Krajcik, J. (2006). Motivation and cognitive engagement in learning environments. En R. K. Sawyer (Edit.). The Cambridge Handbook of the learning science (pp. 475-488). New York: Cambridge University Press.

Brewster, A., \& Bowen, G. (2004). Teacher Support and the School Engagement of Latino Middle and High School Students at Risk of School Failure. Child and Adolescent Social Work Journal, 2l(1), 47-67. https://doi.org/10.1023/B:CASW.0000012348.83939.6b

CAF (2018). Políticas para promover la culminación de la educación media en América Latina y el Caribe. Lecciones desde México y Chile. Recuperado de https://www.r4d.org/wpcontent/uploads/CAF-Promoting-Secondary-School-Retention-in-LAC-SPA.pdf

Fredricks, J. Reschly, A., \& Christenson, S. (2019). Handbook of Student Engagement Interventions. Working with Disengaged Students. San Diego: Academic Press.

Fredricks, J., \& McColskey, W. (2012). The measurement of student engagement: a comparative analysis of various methods and student self-report instruments. En S. Christenson, A. Reschly \& C. Wylie (Eds.) Handbook Research on Student Engagement (pp. 763-782.). New York: Springer.

Fredriscks, J., Blumenfeld, O., \& Paris, A. (2004). School Engagement: Potential of the concept state of the evidence. Review of Educational Research, 74(1), 59- 109. doi: https://doi.org/10.3102/00346543074001059

Garcia-Reid, P., Reid, R., \& Peterson, A. (2005). School Engagement Among Latino Youth in an Urban Middle School Context: Valuing the Role of Social Support. Education and Urban Society, 37(3), 257-275. Doi: https://doi.org/10.1177/0013124505275534

Glasman, D. (2000). Le décrochage scolaire: une question sociale et institutionnelle. VEI Enjeux, 122 , $10-24$.

Recuperado

de https://www.crefe38.fr/IMG/pdf/GLASMAN_VEI2000_decrochagescolaire.pdf

González, M., Tourón, J., \& Gaviria J. (1994). La orientación motivacional intrínseco-extrínseca en el aula: Validación de un instrumento. Bordón, 41(1), 35-50.

Kiefer, S., Alley, K., \& Ellerbrock, C. (2015). Teacher and Peer Support for Young Adolescents' Motivation, Engagement, and School Belonging. RMLE Online, 38 (8), 118. doi: https://doi.org/10.1080/19404476.2015.11641184

Lam, S. Pak, T., \& Ma, W. (2007) Motivating instructional contexts. En P. Zelick (Ed.). Issues in the Psychology of Motivation (pp. 119-136). New York: Nova Science Publishers.

Lam, S., Wong, B., Yang, H. \& Liu, Y. (2012). Understanding student engagement with a contextual model. En S. Christenson, A. Reschly \& C. Wylie (Eds.) Handbook Research on Student Engagement (pp. 403-419). New York: Springer.

Liu, R., Zhen, R., Ding, Y., Liu, Y., Wang, J., Jiang, R., \& Xu, L. (2018). Teacher support and math engagement: roles of academic self-efficacy and positive emotions. Educational Psychology, 38(1), 3-16. doi: https://doi.org/10.1080/01443410.2017.1359238

Lukkarinena, A., Koivukangas, P., \& Seppälä, T. (2016). Relationship between Class Attendance and Student Performance. Procedia - Social and Behavioral Sciences 228, 341 - 347. doi: https://doi.org/10.1016/j.sbspro.2016.07.051

Miranda-Zapata, E., Lara, L., Navarro, J., Saracostti, N., \& de-Toro, X. (2018). Modelling the Effect of School Engagement on Attendance to Classes and School Performance. Revista de Psicodidáctica, 23(2),102-109. doi: https://doi.org/10.1016/j.psicoe.2018.03.001

Nichols, S., \& Dawson, H. (2012). Assessment as a context for student engagement. En S. Christenson, A. Reschly y C. Wylie (Eds.) Handbook Research on Student Engagement (pp. 457-477). New York: Springer.

NSSE (2018). Engagement Insights: Survey Findings on the Quality of Undergraduate Education. Annual Results 2018. Recuperado de http://nsse.indiana.edu/NSSE_2018_Results/pdf/NSSE_2018_Annual_Results.pdf

OCDE (2017). PISA 2015 Results (Volume III). Students' Well-Being. Paris: OCDE Publishing.

Pérez, E., Medrano, L., \& Sanchéz Rosas, J. (2013). El path anlysis: conceptos básicos y ejemplos de aplicación. Revista Argentina de Ciencias del Comportamiento, 5(1), 52-66. 
Reeve, J. (2013). How Students Create Motivationally Supportive Learning Environments for Themselves: The Concept of Agentic Engagement. Journal of Educational Psychology, 105(3), 579-595. doi: http://dx.doi.org/10.1037/a0032690

Rigo, D. (2017). Docentes, tareas y alumnos en la definición del compromiso. Investigando el aula de nivel primario de educación. Educação em Revista, 33, 1-24. doi: http://dx.doi.org/10.1590/0102-4698154275

Rigo, D., \& Donolo, D. (2014). Entre pupitres y pizarrones. Retos en educación primaria: el aprendizaje con compromiso. Educatio Siglo XXI, 32(2), 59-80. Recuperado de http://revistas.um.es/educatio/index

Rigo, D., \& Donolo, D. (2018a). ¿Es posible invertir la forma en que aprendemos y enseñamos? Aderezos para repensar la educación. Revista Innovaciones Educativas, XX(28), 106-119. Recuperado de http://investiga.uned.ac.cr/revistas/index.php/innovaciones/article/view/2135/2501

Rigo, D., \& Donolo, D. (2018b). Construcción y validación. Escala de compromiso hacia las tareas escolares en las clases del nivel primario de educación para estudiantes. Revista Electrónica Psicoespacios, 12(21), 3-22. Recuperado de http://revistas.iue.edu.co/revistasiue/index.php/Psicoespacios/article/view/1079/1331

Rigo, D. Y., \& Donolo, D. (2019). Modelos de ecuaciones estructurales usos en investigación psicológica y educativa. Revista Interamericana De Psicologia/Interamerican Journal of Psychology, 52(3), 345-357. doi: https://doi.org/10.30849/ripijp.v52i3.388

Rigo, D., \& Donolo, D. (2018, no publicado, en evaluación). Compromiso escolar. Estudio de sus dimensiones y razones de desenganche.

Ros, I. (2009). La implicación del estudiante con la escuela. Revista de Psicodidáctica, 14(1), 7992.

Sampieri, R., Collado, C., \& Lucio, P. (2010) Metodología de la Investigación. México: Mc Graw Hill.

Shernoff, D. (2013). Optimal learning environments to promote student engagement. New York: Springer.

Shernoff, D. J., Kelly, S., Tonks, S., Anderson, B., Cavanagh, R., Sinha, S., \& Abdi, B. (2016). Student engagement as a function of environmental complexity in high school classrooms. Learning and Instruction, 43, 52-60. doi: https://doi.org/10.1016/j.learninstruc.2015.12.003

Shernoff, D., Ruzek y, E., \& Sinha, S. (2016). The influence of the high school classroom environment on learning as mediated by student engagement. School Psychology International, 1-18. Doi: 10.1177/0143034316666413

Stipek, D. (1996). Motivation and instruction. In Berliner, D. \& Calfee, R. (Eds.) Handbook of Educational Psychology (pp. 85-113). New York: Simon \& Schuster Macmillan.

Stoner, S., \& Fincham, J. (2012). Faculty Role in Classroom Engagement and Attendance. Am J Pharm Educ, 76(5), 75. doi: https://doi.org/10.5688/ajpe76575

Strati, A. D., Schmidt, J. A., \& Maier, K. S. (2017). El desafío percibido, el apoyo del maestro y la obstrucción del maestro como predictores del compromiso del estudiante. Revista de Psicología de la Educación, 109(1), 131-147. doi: http://dx.doi.org/10.1037/edu0000108

Van Blerkom, M. (2012). Class Attendance in Undergraduate Courses. The Journal of Psychology, 126(5), 487-494. Doi: http://dx.doi.org/10.1080/00223980.1992.10543382

Vigotsky, L. (1988). El desarrollo de los procesos psicológicos superiores. Barcelona: Grijalbo. 